Article

\title{
The Master Transcription Factor $m t f A$ Governs Aflatoxin Production, Morphological Development and Pathogenicity in the Fungus Aspergillus flavus
}

\author{
Zhenhong Zhuang ${ }^{1,+}$, Jessica M. Lohmar ${ }^{1}$, Timothy Satterlee ${ }^{1}$, Jeffrey W. Cary ${ }^{2}$ and \\ Ana M. Calvo ${ }^{1, *}$
}

Received: 7 December 2015; Accepted: 6 January 2016; Published: 20 January 2016

Academic Editor: Shohei Sakuda

1 Department of Biological Sciences, Northern Illinois University, 155 Castle Dr., Dekalb, IL 60115, USA;

xzhzhenhong@163.com (Z.Z.); jlohmar1@niu.edu (J.M.L.); tsatterlee1@niu.edu (T.S.)

2 Food and Feed Safety Research Unit, USDA/ARS, Southern Regional Research Center, New Orleans, LA 70124, USA; jeff.cary@ars.usda.gov

* Correspondence: amcalvo@niu.edu; Tel.: +1-815-753-0451; Fax: +1-815-753-0461

+ Permanent address: Key Laboratory of Pathogenic Fungi and Mycotoxins of Fujian Province, School of Life Sciences, Fujian Agriculture and Forestry University, Fuzhou 350002, China.

\begin{abstract}
Aspergillus flavus produces a variety of toxic secondary metabolites; among them, the aflatoxins (AFs) are the most well known. These compounds are highly mutagenic and carcinogenic, particularly $\mathrm{AFB}_{1}$. A. flavus is capable of colonizing a number of economically-important crops, such as corn, cotton, peanut and tree nuts, and contaminating them with AFs. Molecular genetic studies in A. flavus could identify novel gene targets for use in strategies to reduce AF contamination and its adverse impact on food and feed supplies worldwide. In the current study, we investigated the role of the master transcription factor gene $m t f A$ in $A$. flavus. Our results revealed that forced overexpression of $m t f A$ results in a drastic decrease or elimination of several secondary metabolites, among them $\mathrm{AFB}_{1}$. The reduction in $\mathrm{AFB}_{1}$ was accompanied by a decrease in aflR expression. Furthermore, $m t f A$ also regulates development; conidiation was influenced differently by this gene depending on the type of colonized substrate. In addition to its effect on conidiation, $m t f A$ is necessary for the normal maturation of sclerotia. Importantly, $m t f A$ positively affects the pathogenicity of $A$. flavus when colonizing peanut seeds. AF production in colonized seeds was decreased in the deletion $m t f A$ strain and particularly in the overexpression strain, where only trace amounts were detected. Interestingly, a more rapid colonization of the seed tissue occurred when $m t f A$ was overexpressed, coinciding with an increase in lipase activity and faster maceration of the oily part of the seed.
\end{abstract}

Keywords: aflatoxin; $m t f A$; Aspergillus flavus; secondary metabolism; conidiation; sclerotia; pathogenicity

\section{Introduction}

Species of the genus Aspergillus produce a wide variety of secondary metabolites. Some of these compounds have beneficial bioactive properties, such as antibiotic or anticholesteremic, while others are deleterious, such as mycotoxins [1]. Some of these fungal species are opportunistic plant pathogens. Among them, A. flavus is capable of contaminating agricultural products, particularly oil seeds, at pre-harvest and post-harvest [2]. This is particularly relevant, since A. flavus is notorious for producing highly toxic and carcinogenic mycotoxins. The most well-known are the polyketide-derived compounds known as aflatoxins (AFs), especially $\mathrm{AFB}_{1}$, the most carcinogenic natural compound identified. Ingestion of aflatoxin-contaminated food can result in hepatotoxicity, 
liver cancer, teratogenicity, immunotoxicity and death [3-7]. Searching for novel genetic targets could lead to new strategies to decrease the negative impact of aflatoxin contamination of food supplies.

Considerable progress has been made in the elucidation of the genetic regulatory networks involved in the control of AF production in A. flavus (i.e., [8-13]). The study of mycotoxin production in the model fungus $A$. nidulans has greatly contributed to accelerating the pace in discovering $\mathrm{AF}$ regulators in A. flavus. A. nidulans produces sterigmatocystin (ST), the penultimate precursor in the AF biosynthetic pathway. Both clusters contain aflR homologs, encoding a $\mathrm{Zn}(\mathrm{II})_{2} \mathrm{Cys}_{6}$ transcription factor required for the activation of these gene clusters [14-19]. Recent research using this model organism revealed a new regulatory gene, $m t f A$, encoding a $\mathrm{C}_{2} \mathrm{H}_{2}$ zinc finger transcription factor that affects ST production. $m t f A$ was originally identified through a mutagenesis screening technique designed to reveal novel veA-dependent elements involved in ST regulation in A. nidulans [20]. Both deletion and overexpression of $m t f A$ lead to a reduction of ST production compared to the wild-type strain. Additional analyses showed a broader regulatory role of $m t f A$ in $A$. nidulans, controlling genes involved in the production of other secondary metabolites, besides those involved in ST production [20,21]. $m t f A$ was also identified as a genetic link between secondary metabolism and morphogenesis, positively affecting both asexual and sexual development in the model fungus [20]. Furthermore, this master regulator was also found to perform similar roles in A. fumigatus, an opportunistic animal pathogen that is the most frequent cause of aspergillosis in immunocompromised patients [22]. Absence of $m t f A$ in $A$. fumigatus resulted in alteration in the production of secondary metabolites and conidiation, as well as in a reduction in virulence, as shown in the Galleria mellonella animal model for invasive aspergillosis [22].

A recent transcriptome analysis of $m t f A$ in $A$. nidulans and $A$. fumigatus demonstrated the importance of this regulatory gene as a keystone in the control of the expression of hundreds of genes in these two fungi, extensively affecting secondary metabolite gene clusters. This regulatory scope includes clusters involved in the synthesis of mycotoxins [21], some of them known to be virulence factors during infection (i.e., [23-27]). Genomic analysis indicated that $m t f A$ is conserved in numerous filamentous fungi, particularly among Ascomycetes [20], and it was not found in plant or animal genomes, suggesting that $m t f A$ could be a potential target to control the detrimental effects of numerous pathogenic fungi. In this study, we investigated $m t f A$ in $A$. flavus, revealing a regulatory role in AF production, development and pathogenicity of this agriculturally- and medically-important fungus.

\section{Materials and Methods}

\subsection{Fungal Strains and Growth Conditions}

Aspergillus flavus strains used in the study are listed in Table 1. The strains were cultured on YGT ( $0.5 \%$ yeast extract, $2 \%$ glucose and trace elements, as described in [10]), unless otherwise indicated. The medium was supplemented as required depending on the presence of auxotrophic markers [10,28]. Solid media were prepared by adding $10 \mathrm{~g} / \mathrm{L}$ of agar, except top YGT agar medium (5 g/L). Strains were stored in $30 \%$ glycerol at $-80^{\circ} \mathrm{C}$.

Table 1. Aspergillus flavus strains used in this study.

\begin{tabular}{|c|c|c|}
\hline Strain Name & Related Genotype & Source \\
\hline CA14 & pyrG ${ }^{-}$, niaD ${ }^{-}, \Delta k u 70$ & [29] \\
\hline CA14-pyrG-1 & $n i a D^{-}, \Delta k u 70$ & [29] \\
\hline CA14 pyrG-1-niaD ${ }^{+}$ & prototroph, $\Delta k u 70$ & This study \\
\hline TZZ1 & $\Delta m t f A:: p y r G . n i a D^{-}, \Delta k u 70$ & This study \\
\hline TZZ2 & $\Delta m t f A:: p y r G, \Delta k u 70$ & This study \\
\hline TZZ3 & $\Delta m t f A:: p y r G . n i a D^{-}$, mtfA::niaD ${ }^{+}, \Delta k u 70$ & This study \\
\hline TZZ4 & gpdA(p)::mtfA::trpC(t)::pyrG. niaD ${ }^{-}, \Delta k u 70$ & This study \\
\hline TZZ5 & $g p d A(\mathrm{p}):: m \operatorname{tfA}:: \operatorname{trp} C(\mathrm{t}):: p y r G, \Delta k u 70$ & This study \\
\hline
\end{tabular}




\subsection{Generation of the Deletion, Complementation and Overexpression mtfA Strains}

The $m t f A$ deletion cassette was constructed by fusion PCR as previously described [30]. Both 1.4-kb and flanking untranslated regions (5'UTR and $3^{\prime} \mathrm{UTR}$ ) were PCR amplified from $A$. flavus genomic DNA with primer sets $m t f A$-del-1 and $m t f A$-del-2 and $m t f A$-del-3 and $m t f A$-del-4 respectively (Table 2). The intermediate fragment containing the $A$. fumigatus pyrG marker was amplified from plasmid p1439 [31] by primers $m t f A$-del-5 and -6 (Table 2). The three fragments were PCR fused using primers $m t f A$-del-1 and $m t f A$-del-4. Polyethylene glycol-mediated transformation of $A$. flavus CA14 ( $\triangle$ ku70. pyrG-, niaD-) protoplasts was carried out as described by Cary et al. [32], resulting in the generation of the $m t f A$ deletion strain, TZZ1 ( $\Delta k u 70, \Delta m t f A$. niaD-). Replacement of the entire $m t f A$ coding region with pyrG was confirmed by Southern analysis, as previously described [33]. A prototroph of this deletion strain, $\operatorname{TZZ2}(\Delta k u 70, \Delta m t f A)$, was obtained by a second transformation with the niaD wild-type allele from $A$. fumigatus (Table 1 ).

Table 2. Primers used in this study.

\begin{tabular}{|c|c|}
\hline Primer Name & Sequence \\
\hline$m t f A-$ del-1 & CCCCCATGATTAATGATTGATGGATTTCTGGGCG \\
\hline$m t f A$-del-2 & GGGAGAGCTTGAGCTGTGGAAGGTGGAAGGAT \\
\hline$m t f A-$ del-3 & ACCAAAGCACAAAGACAAGAAACTAAAA \\
\hline$m t f A-d e l-4$ & TACATATGGCATCCTCTCACGAACGTC \\
\hline$m t f A-d e l-5$ & АТССТТССАССТTССАСАGСТСАAGСТСТСССGССТСАААСААТGСТСТTСАССС \\
\hline$m t f A$-del-6 & TTTTAGTTTCTTGTCTTTGTGCTTTGGTGTCTGAGAGGAGGCACTGATGC \\
\hline C-NsiI-S & NNNNNNNATGCATGATTCATCCCCCATGATTAA \\
\hline C-Nsil-A & NNNNNNNATGCATTACATATGGCATCCTCTCAC \\
\hline$m t f A-S$ & GATTCATCCCCCATGATTAA \\
\hline$m t f A-A$ & TACATATGGCATCCTCTCAC \\
\hline O-AscI-S & AAAAAGGCGCGCCATGGATCTCGCCAGCCTTATCACTCC \\
\hline O-NotI-A & AAAAAAAGCGGCCGCTTATACCATGGCGGTGGCGACG \\
\hline gpdA-p & AAGTACTTTGCTACATCCATACTCC \\
\hline niaD-S & ACCGGTCGCCTCAAACAATGCTCTGGCAATGTGAGGCTCCTCCCCAATC \\
\hline niaD-A & GTCTGAGAGGAGGCACTGATGCGGCGATCTCTGGATCAATACGACCGAC \\
\hline qPCR-Afla_18S_F & TGATGACCCGCTCGGCACCTTACGAGAAATCAAAGT \\
\hline qPCR-Afla_18S_R & GGCCATGCACCACCATCCAAAAGATCAAGAAAGAGC \\
\hline qPCR-Afla_ver1_F & GCGGAGAAAGTGGTTGAACAGATC \\
\hline qPCR-Afla_ver1_R & CAGCGAACAAAGGTGTCAATAGCC \\
\hline qPCR-Afla_brlA_F & TATCCAGACATTCAAGACGCACAG \\
\hline qPCR-Afla_brlA_R & GATAATAGAGGGCAAGTTCTCCAAAG \\
\hline qPCR-Afla_aflR_F & GCAACCTGATGACGACTGATATGG \\
\hline qPCR-Afla_aflR_R & TGCCAGCACCTTGAGAACGATAAG \\
\hline qPCR-Afla_mtfA_F & AGTGTGGCCTCGTACTCTTCGCCGGTTGAATCCTC \\
\hline qPCR_Afla_mtfA_R & GTCGTGGTTCTGTTGGTAGGGTGCCGAGCTGGAAG \\
\hline
\end{tabular}

The $m t f A$ complementation strain was constructed by transforming the $m t f A$ deletion mutant with the $m t f A$ wild-type allele. The complementation $m t f A$ vector was generated as follows: a DNA fragment containing the coding region of $m t f A$ and $1.4 \mathrm{~kb} 5^{\prime}$ and $3^{\prime}$ UTRs was PCR amplified with primers C-NsiI-S and C-NsiI-A (Table 2) and digested by NsiI. The digested PCR fragment was cloned into pSD52 plasmid previously digested with the same enzyme. pSD52 harbored the selection marker niaD from $A$. fumigatus. The resulting recombinant plasmid, pSD52.2- $m t f A$, was used to transform TZZ1 $(\Delta k u 70, \Delta m t f A$. niaD-), obtaining the $m t f A$ complementation prototroph TZZ3 (Table 1). The presence of pSD50.2- $m t f A$ in TZZ3 was confirmed by diagnostic PCR using primers $m t f A$-del-1 and $m t f A$-del-4.

In order to obtain the $m t f A$ overexpression strain $(\mathrm{OE} m t f A)$, a fragment containing the $m t f A$ coding region was PCR amplified from CA14 (Table 1) genomic DNA with primers O-AscI-S and O-NotI-A (Table 2). The fragment was digested with AscI and NotI and ligated into pTDS1 previously digested with the same enzymes. pTDS1 contains the A. nidulans gpdA promoter and $\operatorname{trp} C$ terminator. The resulting vector, pTDS1- $m$ tf $A$, was then transformed in CA14, generating TZZ4 (Table 1). Integration of pTDS1-mtfA into TZZ4 was verified by diagnostic PCR with primers gpdAp 
and O-Not1-A. TZZ4 was then transformed with the wild-type allele of niaD from A. fumigatus, obtaining the prototroph TZZ5 (Table 1).

\subsection{Aflatoxin $B_{1}$ Analysis}

$\mathrm{AFB}_{1}$ was extracted with chloroform from fungal cultures grown as specified in each case. Extracts were allowed to dry and then resuspended in $500 \mu \mathrm{L}$ of $\mathrm{CHCl}_{3}$ before $25 \mu \mathrm{L}$ of each extract was fractionated on a silica gel thin-layer chromatography (TLC) using a toluene-ethyl acetate-formic acid (5:4:1, $v / v / v)$ solvent system. TLC plates were sprayed with $\mathrm{AlCl}_{3}\left(15 \%\right.$ in ethanol) and baked at $80{ }^{\circ} \mathrm{C}$ for $10 \mathrm{~min}$ to intensify $\mathrm{AFB}_{1}$ fluorescence upon exposure to long-wave UV fluorescence at $375 \mathrm{~nm}$. Commercial $\mathrm{AFB}_{1}$ (Sigma-Aldrich, St. Louis, MO, USA) was used as the standard.

\subsection{Morphological Analysis}

Conidia (10 $10^{6}$ spores $/ \mathrm{mL}$ ) of Aspergillus flavus wild-type (CA14 pyrG-1-niaD+), $\Delta m t f A$, (TZZ2) complementation (TZZ3) and OEmtfA (TZZ5) strains were added to $5 \mathrm{~mL}$ of top-agar $(0.4 \%)$ that was subsequently poured onto the surface of YGT agar medium. Seven-millimeter diameter cores were collected during a time-course experiment. The cores were homogenized, and conidia were quantified with a hemocytometer (Hausser Scientific, Horsham, PA, USA) under a Nikon Eclipse E-400 bright-field microscope (Nikon Inc., Melville, NY, USA).

The effect of $m t f A$ on sclerotial production was also assessed under the same experimental conditions. Sixteen millimeter cores were sprayed with $70 \% \mathrm{EtOH}$ in order to improve visualization of sclerotia. Micrographs were taken using a Leica MZ75 dissecting microscope coupled to a Leica DC50LP camera (Leica Microsystems Inc., Buffalo Grove, IL, USA).

\subsection{Gene Expression Analysis}

Strains were incubated in liquid stationary cultures in Petri dishes containing $30 \mathrm{~mL}$ of liquid YGT. Plates inoculated with conidia $\left(10^{6}\right.$ spores $\left./ \mathrm{mL}\right)$ of $A$. flavus wild-type, $\triangle m t f A$, complementation and $\mathrm{OE} m t f A$ strains were incubated at $30^{\circ} \mathrm{C}$ in the dark. Total RNA was extracted from lyophilized mycelia using TRIsure (Bioline, Taunton, MA, USA) reagent and an RNeasy Plant Mini Kit (Qiagen, Valencia, CA, USA) according to the manufacturer instructions. Gene expression analysis was performed by qRT-PCR. First, five micrograms of total RNA were treated with RQ1 RNase-Free Dnase (Promega, Madison, WI, USA). cDNA was synthesized with Moloney murine leukemia virus (MMLV) reverse transcriptase (Promega). qRT-PCR was performed with the Applied Biosystems 7000 Real-Time PCR System using SYBR green dye for fluorescence detection. A. flavus $18 \mathrm{~S}$ ribosomal gene expression was used as the reference, and the relative expression levels were calculated using the $2^{-\Delta \Delta C T}$ method [34]. The primer pairs used are indicated in Table 2.

\subsection{Pathogenicity Study}

The NC94022 Virginia peanut line was utilized to examine the possible role of $m t f A$ on seed infection as previously described [13] with minor modifications. All seeds used in this experiment were shelled, separated, the embryos removed and weighed out to approximately $0.25 \mathrm{~g}$ to $0.35 \mathrm{~g}$. Each cotyledon was surface sterilized with 10\% Clorox bleach for approximately $1 \mathrm{~min}$ and then washed with sterile $\mathrm{ddH}_{2} \mathrm{O}$ twice. The viable cotyledons were then dried and placed on sterile glass Petri dish plate (12 peanut cotyledons per plate). Individual cotyledons were inoculated on the adaxial surface with $50 \mu \mathrm{L}$ of water containing approximately $2.0 \times 10^{6}$ spores $/ \mathrm{mL}$. The cultures were incubated for 7 days at $30^{\circ} \mathrm{C}$ in the dark.

Ergosterol content is used as an indicator of fungal burden in infected seeds [13,17]. After 4 and 7 days of incubation at $30^{\circ} \mathrm{C}$, four infected peanut cotyledons were collected from each culture, ground in liquid nitrogen and extracted with a 4-mL solution of chloroform-methanol in a 2:1 ratio overnight at room temperature. The extraction mixtures were then filtered through sterile Miracloth into a $50-\mathrm{mL}$ beaker. The extracts were allowed to evaporate and re-suspended in $3 \mathrm{~mL}$ of the 
same extraction mixture. One milliliter of each sample was filtered through a 0.2-micron filter and placed into a 1-mL HPLC vial for analysis. Twenty-five microliters of each sample were injected into a Waters 1525 HPLC system (Waters, Milford, MA, USA) equipped with a binary pump and a Waters 717 autosampler (Waters, Milford, MA, USA). HPLC separation was carried out at $50{ }^{\circ} \mathrm{C}$ on a Phenomenex C18 $4.6 \times 25 \mathrm{~mm}$ 5-micron analytical column attached to a column guard and $100 \%$ HPLC grade methanol as the mobile phase at a flow rate of $1.0 \mathrm{~mL} / \mathrm{min}$. UV detection at $282 \mathrm{~nm}$ was performed with a Waters 2487 Dual $\lambda$ Absorbance Detector (Waters, Milford, MA, USA). HPLC-grade ergosterol (Sigma-Aldrich, St. Louis, MO, USA) was used as standard reference to determine ergosterol concentration in the samples. The experiment was carried out with 3 replicates.

$\mathrm{AFB}_{1}$ content in infected peanut cotyledons was evaluated after 7 days of incubation at $30^{\circ} \mathrm{C}$. Four cotyledons were collected from each culture and ground in liquid nitrogen. Then, $12 \mathrm{~mL}$ of sterile $\mathrm{ddH}_{2} \mathrm{O}$ were added, and the suspensions were placed in sterile 50-mL beakers to which $6 \mathrm{~mL}$ of acetone was added. The beakers were then placed on a rotary platform. After $1 \mathrm{~h}$, each sample was filtered through Whatman paper and placed in a 50-mL Falcon tube to which $17 \mathrm{~mL}$ of methylene chloride were added. The content of each tube was mixed by inversion and centrifuged at $4000 \mathrm{rpm}$ for $5 \mathrm{~min}$. The bottom organic layer was collected, passed through a filter with granulated sodium sulfate to absorb remaining water and allowed to evaporate overnight. The dried extracts were re-suspended in $300 \mu \mathrm{L}$ of acetone and transferred into 1.5-mL Eppendorf tubes. The extracts were again allowed to evaporate overnight and re-suspended in $100 \mu \mathrm{L}$ of acetone. Twenty-five microliters of each extract were then separated by TLC as described above. This experiment was carried out in triplicate.

Conidial production on the infected seeds was examined after 7 days of incubation at $30{ }^{\circ} \mathrm{C}$ in the dark. In this case, four cotyledons were harvested from each culture and placed into 1.5-mL Eppendorf tubes. One milliliter of $\mathrm{ddH}_{2} \mathrm{O}$ was added to each tube and vortexed for $1 \mathrm{~min}$. Conidia were quantified with a hemocytometer (Hausser Scientific, Horsham, PA, USA) as detailed above.

\subsection{Hydrolytic Activity Analysis}

Aspergillus flavus produces a variety of extracellular hydrolytic enzymes required for successful colonization and subsequent toxin contamination of crops [35,36]. For this reason, we also investigated whether $m t f A$ affects hydrolytic activity in A. flavus, specifically lipase, protease and amylase activity.

\subsubsection{Lipase Activity}

Lipase activity was examined as previously described [37] with minor modifications. Briefly, $100 \mu \mathrm{L}$ of conidial suspensions $\left(10^{6}\right.$ spores $\left./ \mathrm{mL}\right)$ of the $A$. flavus wild-type, $\Delta m t f A$, complementation and OEmtfA strains were inoculated on $10 \mathrm{~mL}$ of tributyrin agar (per liter: $3 \mathrm{~g}$ yeast extract, $5 \mathrm{~g}$ peptone, $10 \mathrm{~mL}$ tributyrin, $10 \mathrm{~g}$ agar, $\mathrm{pH}$ 7.5) in test tubes with 6 replicates per strain. The inoculated cultures were incubated at $30^{\circ} \mathrm{C}$ in the dark. Zones of degradation were measured after 4 to 7 days of incubation.

\subsubsection{Protease Activity}

To assess whether $m t f A$ plays a role influencing protease activity in $A$. flavus, the strains $\left(10^{6}\right.$ spores $/ \mathrm{mL}$ ) were inoculated into $500 \mathrm{~mL}$ of PMS (Peptone Minimal Salts) broth (per $1 \mathrm{~L}: 50 \mathrm{~g}$ peptone, $3 \mathrm{~g}\left(\mathrm{NH}_{4}\right)_{2} \mathrm{SO}_{4}, 10 \mathrm{~g} \mathrm{~K}_{2} \mathrm{HPO}_{4}, 2 \mathrm{~g} \mathrm{MgSO} \cdot 7 \mathrm{H}_{2} \mathrm{O}, 1 \mathrm{~mL}$ of trace elements, $\mathrm{pH}$ 5.2) and incubated at $37^{\circ} \mathrm{C}$ at $250 \mathrm{rpm}$ for $24 \mathrm{~h}$. To induce protease activity, approximately $1 \mathrm{~g}$ of mycelia was shifted into $25 \mathrm{~mL}$ of liquid 0.01\% GMM (Glucose Minimum Medium) containing $8 \mathrm{mg} / \mathrm{mL}$ of bovine serum albumin (BSA) and allowed to further incubate at $250 \mathrm{rpm}$ at $30{ }^{\circ} \mathrm{C}$. Fungal supernatants were collected after 24,48 and $72 \mathrm{~h}$ of incubation and filtered through $0.2-\mu \mathrm{m}$ low-protein-binding filters. Protease activity was measured by an azocasein assay as previously described [38]. 


\subsubsection{Amylase Activity}

Amylase activity was evaluated by TLC as described by Duran et al. [38] with some modifications. The strains were inoculated in a 1-L flask $\left(10^{6}\right.$ spores $\left./ \mathrm{mL}\right)$ containing $500 \mathrm{~mL}$ PMS broth. The cultures were incubated at $37^{\circ} \mathrm{C}$ for $24 \mathrm{~h}$ at $250 \mathrm{rpm}$. After incubation, mycelia were washed three times with sterile $\mathrm{ddH}_{2} \mathrm{O}$ before shifting approximately $1 \mathrm{~g}$ of mycelia into a 125-mL flask containing $25 \mathrm{~mL}$ of an amylase-inducing medium (GMM, with $1 \%$ starch as the carbon source instead of glucose). The cultures were incubated at $30^{\circ} \mathrm{C}$ at $250 \mathrm{rpm}$. After 24,48 and $72 \mathrm{~h}$ of incubation, approximately $100 \mu \mathrm{L}$ of fungal supernatant were mixed with $100 \mu \mathrm{L}$ of $0.5 \%$ maltoheptose solution and incubated at $40{ }^{\circ} \mathrm{C}$ for $18 \mathrm{~h}$. The reaction was stopped by heating the mixture to $100^{\circ} \mathrm{C}$ for $5 \mathrm{~min}$. Five microliters of the degradation products were loaded onto a Silica Pre-Coated Polygram Sil G/UV 254 TLC plate (Macherey-Nagel, Bethlehem, PA, USA) and compared to $5 \mu \mathrm{L}$ of glucose, maltose and maltotriose standards $(1 \mathrm{mg} / \mathrm{mL})$. The TLC plate was then developed in an isopropanol-water-ammonium hydroxide (70:30:10, $v / v)$ solvent system. After development, the TLC plate was sprayed with $30 \%$ sulfuric acid and dried before being charred at $100{ }^{\circ} \mathrm{C}$ for $5 \mathrm{~min}$ and photographed with a Sony Cybershot DSC-W120 camera (Sony, New York, NY, USA).

\subsection{Statistical Analysis}

Quantitative data were analyzed using ANOVA (analysis of variance) in conjunction with Tukey's test. Differences among mean comparisons of the A. flavus strains were considered significant if the $p$-value was less than $0.05(p<0.05)$.

\section{Results}

3.1. mtfA Affects $A F B_{1}$ Biosynthesis as well as the Production of Other Unknown Secondary Metabolites in A. flavus

In order to elucidate the role of $m t f A$ (Accession Number AFLA_091490) in A. flavus wild-type, deletion $(\triangle m t f A)$ (TZZ2), complementation (TZZ3) and overexpression (OEmtfA) (TZZ5) strains were generated as specified in the Materials and Methods Section. The strains were verified by Southern blot analysis or diagnostic PCR (Figures 1 and 2). In addition, $m t f A$ expression levels in wild-type, $\triangle m t f A$, complementation and OEmtfA strains were also examined (Figures $1 \mathrm{E}$ and $2 \mathrm{C}$ ). As predicted, the $\triangle m t f A$ strain did not show $m t f A$ expression. Expression of $m t f A$ in the OE $m t f A$ strain was greater than in the wild-type strain, indicating that the overexpression cassette was functional.

A

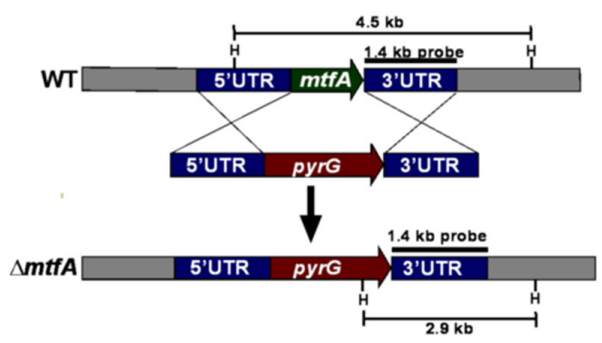

C

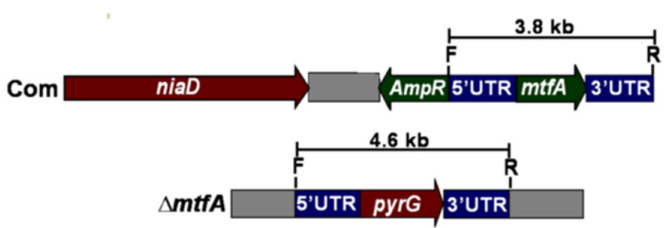

B

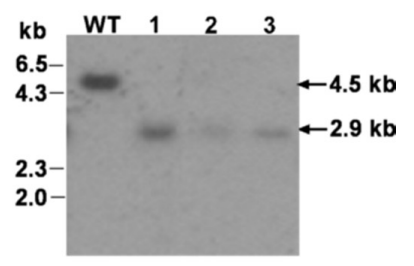

D

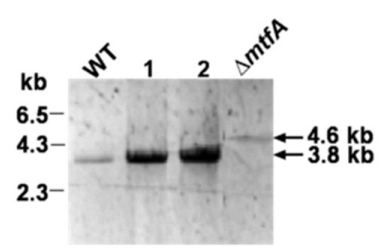

Figure 1. Cont. 


\section{E}

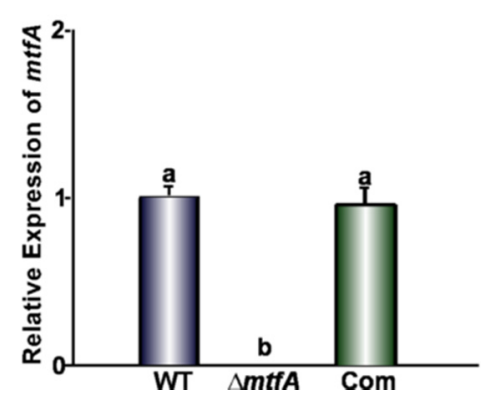

Figure 1. Generation and verification of $\triangle m t f A$ and $m t f A$ complementation strains. (A) Diagram showing the replacement of $m t f A$ with the $p y r G$ marker by a double-crossover event. HindIII restrictions sites and the probe template are shown. (B) X-ray image of the Southern blot analysis confirming the deletion of $m t f A$. Genomic DNA samples were digested with HindIII $(\mathrm{H})$. The Expected band sizes were $4.5 \mathrm{~kb}$ for the wild-type and $2.9 \mathrm{~kb}$ for $\Delta m t f A$. All mutant strains presented the same phenotype; transformant 1 (TZZ2) was used for further studies. (C) Linearized representation of the complementation plasmid (pSD2.2-mtfA). (D) Results of diagnostic PCR, confirming the insertion of the complementation plasmid carrying the $m t f A$ wild-type allele in the $\Delta m t f A$ strain, using primers C-Nsil-S and C-NsiI-A (Table 2), indicated as F and R, respectively in (C). The wild-type strain and deletion $m t f A$ mutant were used as controls. The presence of a $3.8-\mathrm{kb}$ band shows proper integration in the $m t f A$ locus, while a $4.6-\mathrm{kb}$ band indicates the altered locus in $\triangle m t f A$. (E) Expression analysis of $m t f A$ by qRT-PCR with primers qPCR-Afla_mtfA_F and qPCR-Afla_mtfA_R (Table 2). The relative expression was calculated using the $2^{-\Delta \Delta C T}$ method, as described by Livak and Schmittgen [34]. The expression of $18 \mathrm{~S}$ rRNA was used as an internal reference. Values were normalized to the expression levels in the wild-type, considered as one. Error bars represent the standard errors. Different letters above the bars represent significantly different values $(p \leqslant 0.05)$.
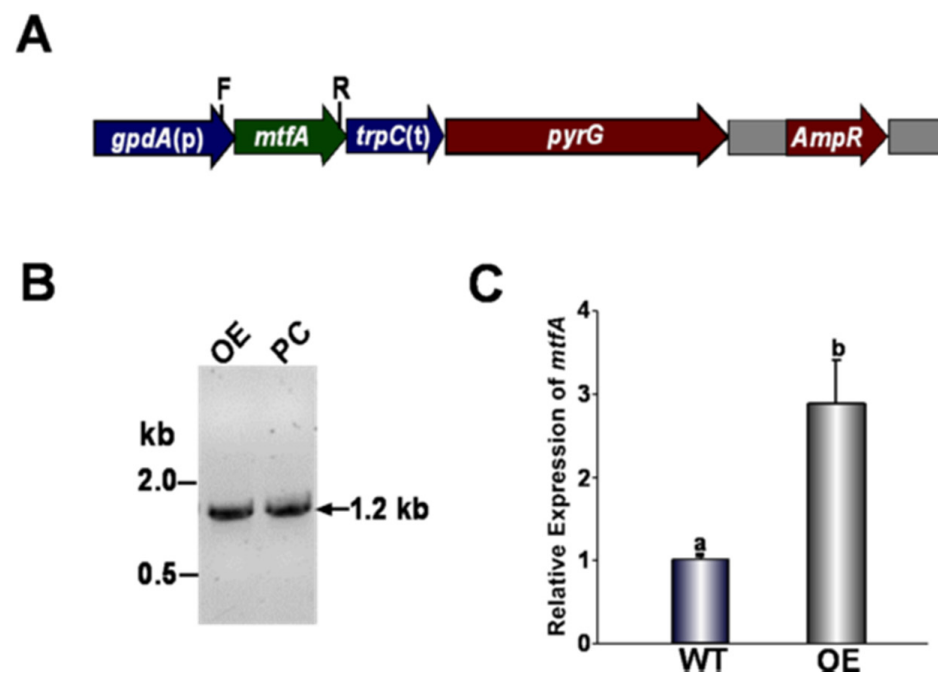

Figure 2. Generation and verification of $m t f A$ overexpression strain (OEmtfA). (A) Linearized representation of the pTDS1- $m$ tfA plasmid. Primers gpdA-p and O-NotI-A, shown as F and R, respectively, were used for PCR amplification. (B) A 1.2-kb PCR product indicating the integration of the overexpression plasmid into the genome. The overexpression plasmid was used as a positive control (PC). (C) Expression analysis of $m t f A$ by qRT-PCR indicating greater accumulation of $m t f A$ transcripts in the $m t f A$ overexpression strain compared to that of the wild-type. The relative expression was calculated using the $2^{-\Delta \Delta C T}$ method, as described by Livak and Schmittgen [34]. Expression of $18 \mathrm{~S}$ rRNA was used as an internal reference. Values were normalized to the expression levels in the wild-type, considered as one. Error bars represent the standard errors. Different letters above the bars represent significantly different values $(p \leqslant 0.05)$. 
$m t f A$ was previously shown to regulate the production of several secondary metabolites, including ST, penicillin and terriquinone A in A. nidulans [20] and gliotoxin in A. fumigatus [22]. Furthermore, transcriptome analyses show a broad effect of $m t f A$ affecting many genes in the genome of these two fungi [21]. To assess whether $m t f A$ was necessary for $A$. flavus secondary metabolism, we specifically examine its possible role in the regulation of $\mathrm{AFB}_{1}$ biosynthesis. Our TLC analyses revealed that overexpression of $m t f A$ dramatically reduces $\mathrm{AFB}_{1}$ production compared to the control strains (Figure 3A). The observed reduction was accompanied by a drastic reduction in aflR expression, as well as a reduction in ver- 1 expression, commonly used as an indicator of AF cluster activation (Figure $3 \mathrm{~B}, \mathrm{C}$ ). The absence of the $m t f A$ did not have a significant effect on $\mathrm{AFB}_{1}$ production under the conditions tested (data not shown). It was also noted by TLC analysis that other unknown metabolites were absent or produced at lower levels in the OEmtfA strain, while they were present or produced at higher amounts in the wild-type strain (Figure 3A).

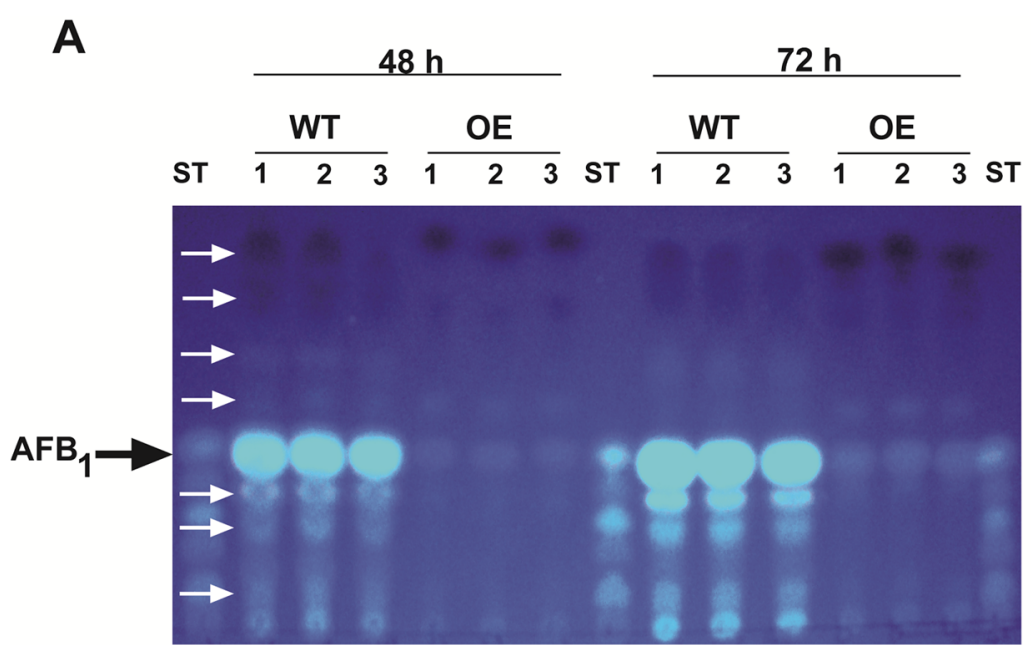

B

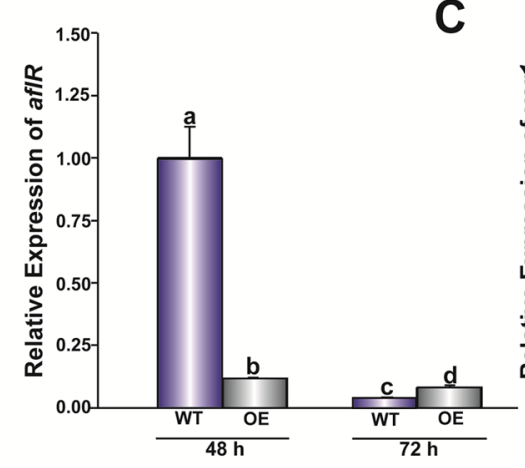

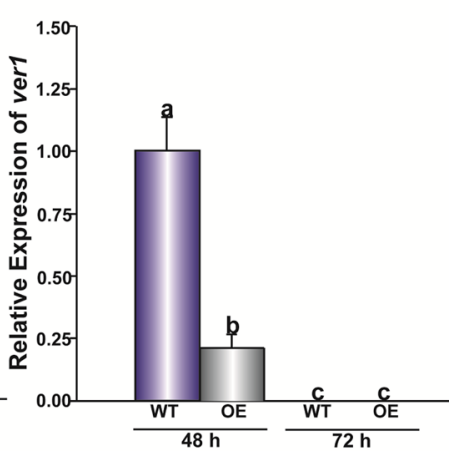

Figure 3. Role of $m t f A$ in $\mathrm{AFB}_{1}$ production. (A) TLC analysis of YGT liquid stationary cultures of wild-type (WT) CA14 pyrG-1-niaD+ and overexpression strain (OE) (TZZ5) after $48 \mathrm{~h}$ and $72 \mathrm{~h}$ of incubation. Unknown metabolites are indicated by white arrows. ST (sterigmatocystin) indicates the $\mathrm{AFB}_{1}$ standard. Expression analysis of aflR (B) and ver1 (C). The relative expression was calculated using the $2^{-\triangle \Delta C T}$ method, as described by Livak and Schmittgen [34]. The expression of $18 \mathrm{~S}$ rRNA was used as an internal reference. Values were normalized to the expression levels in the wild-type, considered as one. Error bars represent the standard errors. Different letters above the bars represent significantly different values $(p \leqslant 0.05)$.

\subsection{Morphological Development Is Regulated by $m t f A$ in A. flavus}

In order to determine if $m t f A$ plays a role-regulating conidiation, the strains were grown on top-agar inoculated cultures and in liquid stationary cultures. An increase in conidial production was observed in the $\triangle m t f A$ strain when compared to the isogenic controls in both types of cultures, 
whereas the OEmtfA strain exhibited a statistically-significant decrease in conidial production (Figure 4). This result was concomitant with the expression pattern of $b r l A$, a key gene in the central regulatory pathway known to regulate conidiation in Aspergillus $[39,40]$. In our experiment, brlA transcription levels were significantly higher in the $\Delta m t f A$ strain at both time points measured, whereas they were reduced in the OEmtfA strain compared to the controls (Figure 4C). A. flavus also differentiates forming resistant structures termed sclerotia. Top-agar inoculated cultures were examined after 14 days of incubation. All of the strains were able to produce sclerotia under the experimental conditions assayed; however, the size of these structures was notably smaller in the $\Delta m t f A$ mutant with respect to the controls (Figure 5).

A

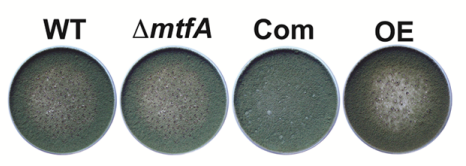

\section{C}

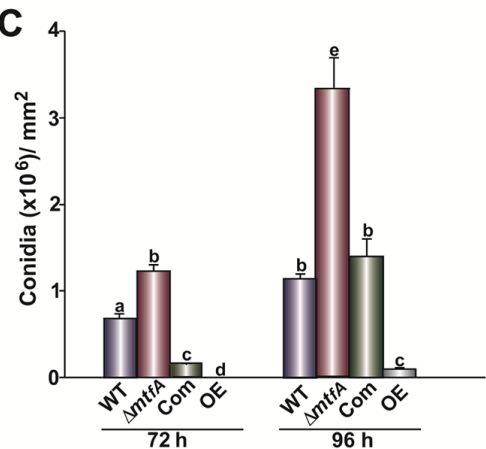

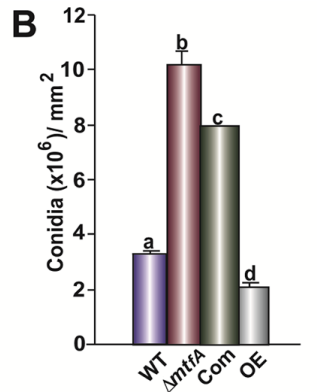

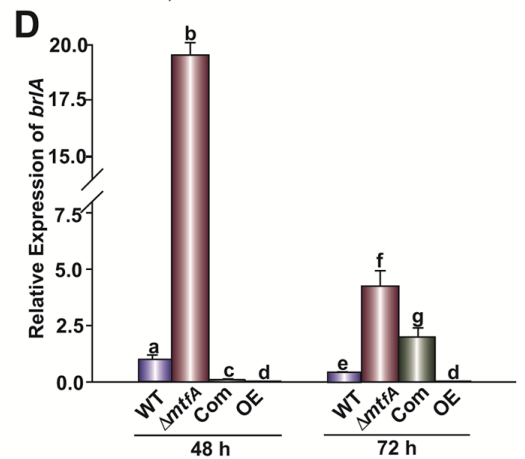

Figure 4. $m t f A$ negatively regulates A. flavus asexual development. (A) Photographs of YGT top-agar inoculated cultures of $A$. flavus wild-type (WT), $\Delta m t f A$, complementation (Com) and $m t f A$ overexpression (OE). Strains were incubated for seven days at $30{ }^{\circ} \mathrm{C}$. (B) Conidial quantifications of cultures shown in (A). (C) Conidial quantifications of liquid stationary cultures after 48 and $72 \mathrm{~h}$ of incubation. (D) Relative expression levels of $b r l A$. The error bars represent the standard errors. Different letters above the bars represent significantly different values $(p \leqslant 0.05)$.
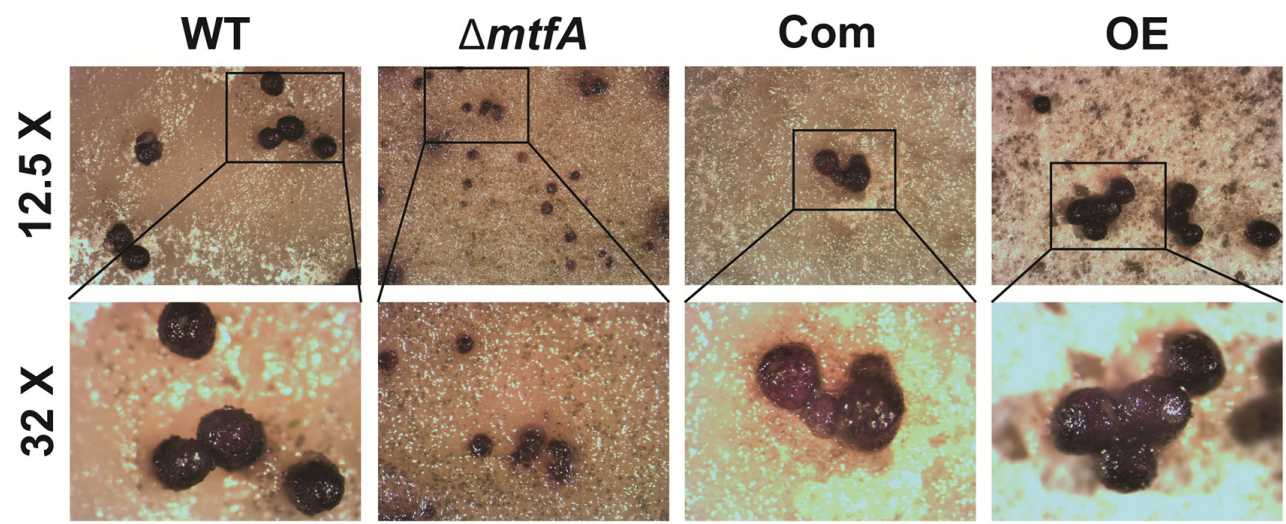

Figure 5. $m t f A$ is necessary for the normal size of sclerotia. Micrographs ( $12.5 \times$ and $32 \times$ magnification) of YGT-top agar inoculated cultures after 14 days of incubation. Cultures were washed with $70 \% \mathrm{EtOH}$ to improve the visualization of sclerotia. 


\section{3. $m t f A$ Is Necessary for Normal Virulence of A. flavus Peanut Infections}

Aspergillus flavus is widely known as an opportunistic pathogen of economically-important oil seed crops. $m t f A$ was previously shown to be necessary for virulence in $A$. fumigatus using an animal infection model [22]. However, the importance of $m t f A$ in plant tissue colonization and mycotoxin contamination of this substrate has not yet been investigated. In our experiment, surfaced-sterilized viable peanuts seeds were inoculated with $A$. flavus wild-type, $\Delta m t f A$, complementation and OEmt $f A$ strains. Our results revealed a significant decrease in conidiation in the $\triangle m t f A$ infected seeds after seven days of incubation with respect to the wild-type levels (Figure 6A,B). We also examined ergosterol content in infected seeds as an indicator of fungal load. Although conidiation was reduced in the absence of $m t f A$, levels of fungal biomass were similar in the $m t f A$ deletion mutant with respect to the controls. Interestingly, ergosterol levels also indicated that overexpression of $m t f A$ resulted in faster colonization of seed tissue compared to the wild-type (Figure 6C). This was accompanied by a more notable softening of the seeds colonized by the OEmtfA strain compared to seeds infected by the other A. flavus strains tested (data not shown).

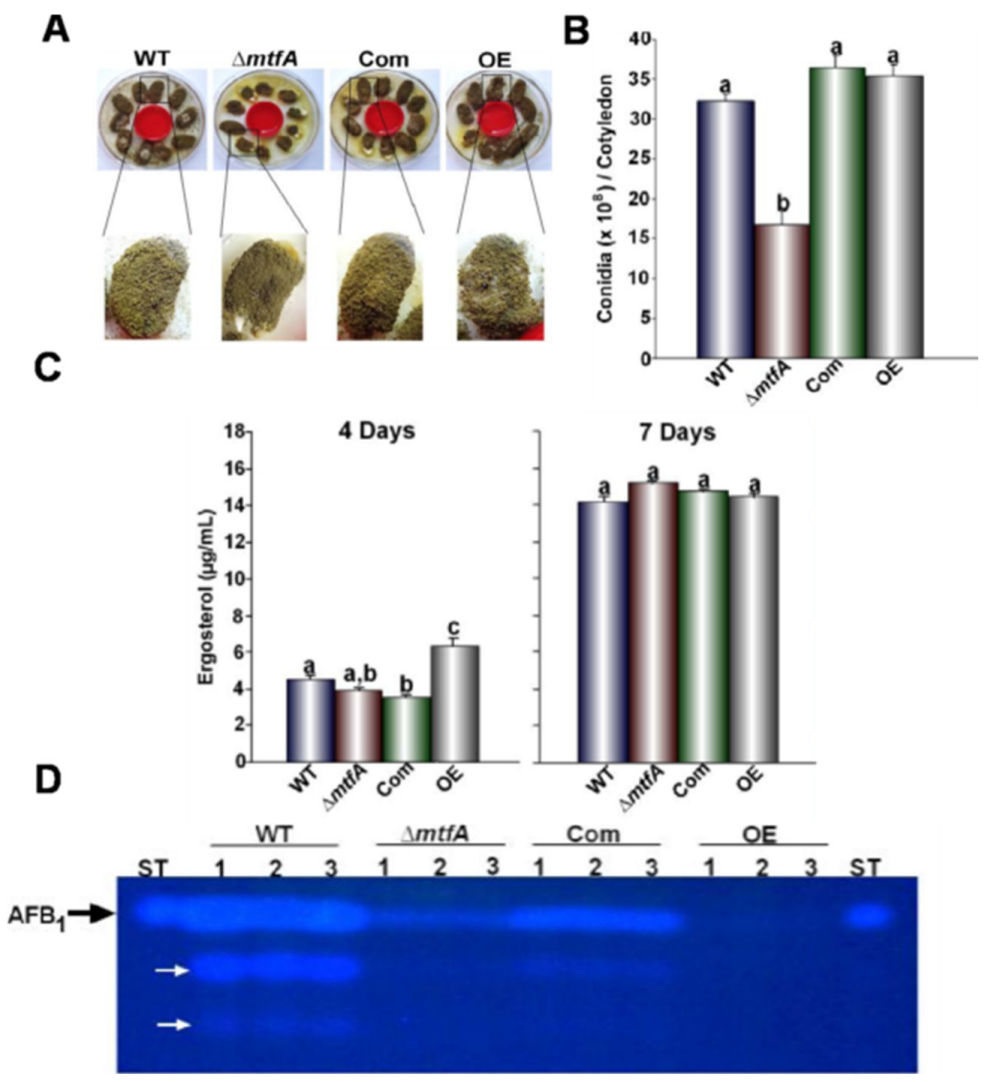

Figure 6. Role of $m t f A$ in the A. flavus pathogenicity of peanut seeds. (A) Photographs of the NC94022 viable peanut line infected with the A. flavus strains after seven days of incubation. (B) Quantification of conidia present in infected peanut cotyledons in (A). (C) Ergosterol content in infected seeds after four and seven days of incubation. (D) TLC analysis of $\mathrm{AFB}_{1}$ levels present in infected peanut cotyledons. White arrows indicate unknown secondary metabolites. Error bars represent the standard error. Different letters above the bars represent significantly different values $(p \leqslant 0.05)$.

$\mathrm{AFB}_{1}$ content of infected peanuts seeds was also analyzed (Figure 6D). Importantly, a decrease in $\mathrm{AFB}_{1}$ levels was observed in seeds infected with the $\triangle m t f A$ and OEmtfA strains with respect to those infected with the isogenic controls. This reduction in AFB1 production was more pronounced when $m t f A$ was overexpressed. 


\section{4. mtfA Positively Affects Lipase and Protease Activity While It Is Dispensable for Normal Amylase Activity}

Due to the effect observed by $m t f A$ on $A$. flavus virulence on peanut seeds, we investigated whether this regulator mediates hydrolytic activity that could be associated with seed colonization by this fungus. We first evaluated whether lipase activity is $m t f A$-dependent. The wild-type, $\Delta m t f A$, complementation and OEmtfA strains were grown on a medium containing the short chain fatty acid tributyrin. Our analysis revealed a slight, but statistically-significant decrease in the zones of degradation in the $\triangle m t f A$ culture with respect to the controls at all time points assayed, indicating a reduction in lipase activity in the absence of $m t f A$ (Figure 7). In addition, an increase in lipase activity was observed after seven days of incubation in the OEmtfA strain. Protease activity was also assessed using bovine serum albumin as the substrate. In this experiment, protease activity was also slightly downregulated in the absence of $m t f A$ (Figure 8 ). We also analyzed the effect of $m t f A$ on amylase activity in $A$. flavus. Our results indicated that this enzymatic activity is not affected by this regulatory gene (Figure 9).

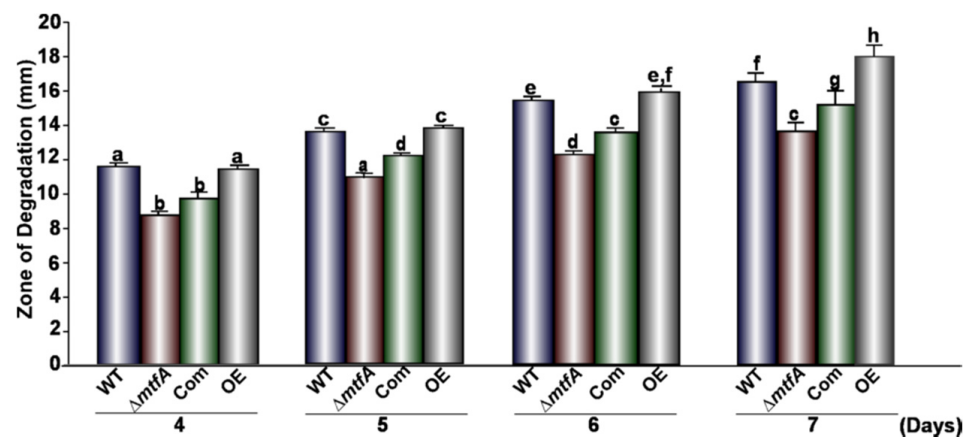

Figure 7. $m t f A$ is necessary for normal lipase activity in A. flavus. The wild-type, $\Delta m t f A$, complementation and overexpression strains were grown on tributyrin agar where zones of degradation were measured (mm) after 4, 5, 6 and 7 days of incubation. Error bars represent the standard error. The experiment was carried out with six replicates. Different letters above the bars represent significantly different values $(p \leqslant 0.05)$.

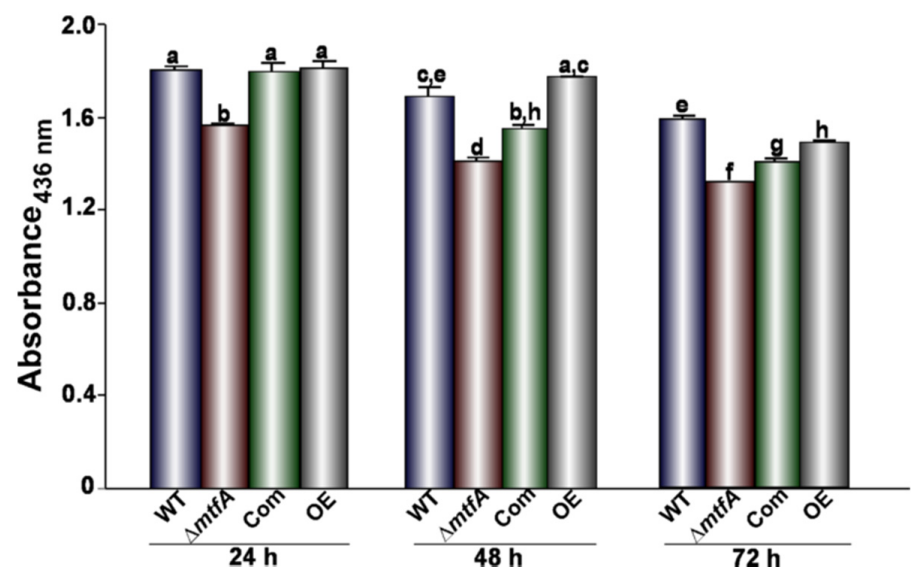

Figure 8. Effect of $m t f A$ on protease activity. The A. flavus strains were grown in PMS broth at $37^{\circ} \mathrm{C}$ and $250 \mathrm{rpm}$ for $24 \mathrm{~h}$ before being shifted into liquid $0.1 \%$ GMM containing $8 \mathrm{mg} / \mathrm{mL}$ of BSA. Fungal supernatants were collected after 24,48 and $72 \mathrm{~h}$ of incubation at $30{ }^{\circ} \mathrm{C}$ and at $250 \mathrm{rpm}$. Protease production was evaluated via an azocasein assay. Absorbance was read at $436 \mathrm{~nm}$. The experiment was carried out with three replicates. Error bars indicate the standard error. Different letters above the bars represent significantly different values $(p \leqslant 0.05)$. 


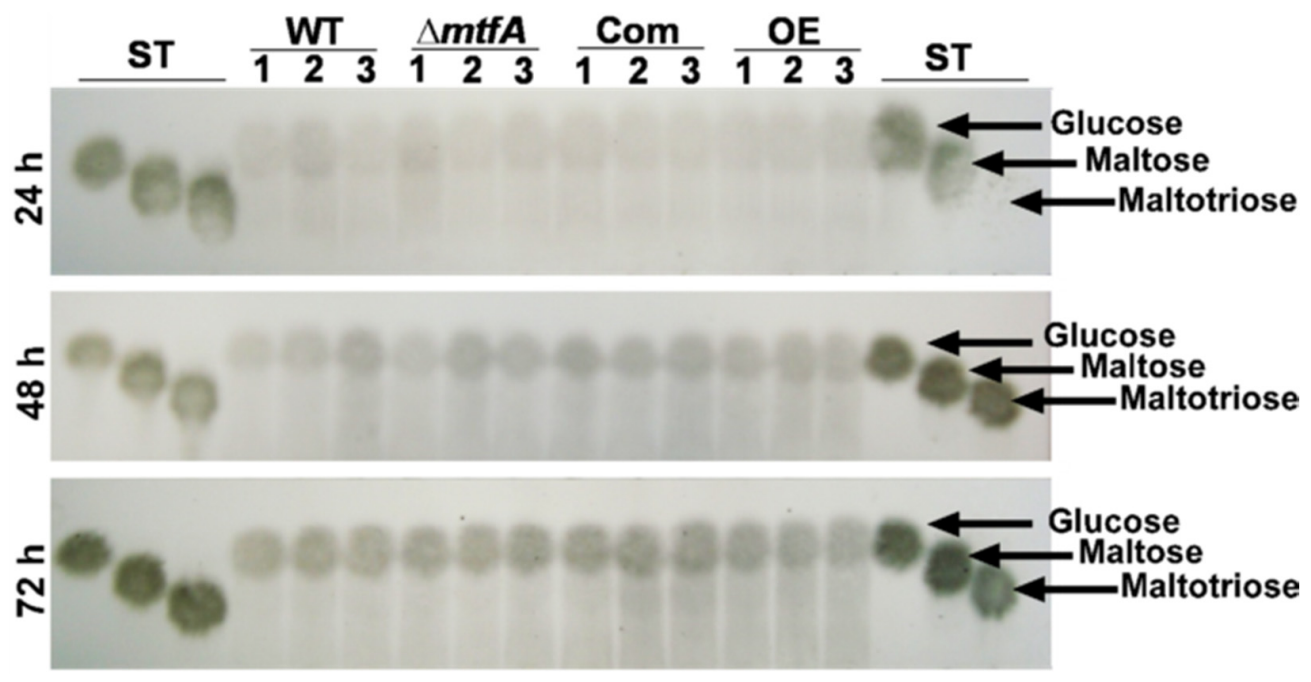

Figure 9. $m t f A$ is dispensable for amylase production in A. flavus. The wild-type, $\Delta m t f A$, complementation and overexpression strains were grown in PMS broth at $37^{\circ} \mathrm{C}$ and $250 \mathrm{rpm}$ for $24 \mathrm{~h}$ before being shifted into minimum medium containing $1 \%$ starch as the carbon source and incubated at $250 \mathrm{rpm}$. Fungal supernatants were collected after 24, 48 and $72 \mathrm{~h}$ of incubation at $30{ }^{\circ} \mathrm{C}$, and amylase production was analyzed by using maltoheptose as the substrate. TLC analysis of degradation products was performed using glucose, maltose and maltotriose standards (ST) as the reference. The experiment was carried out with three replicates.

\section{Discussion}

Aspergillus flavus is able to colonize economically-important crops, such as peanut, maize, cotton, tree nut and other oil seed crops. This fungus produces numerous secondary metabolites, including the carcinogenic and mutagenic compounds called aflatoxins (AFs). While consumption of AF-contaminated foods and feeds can have serious adverse health consequences in developing countries, in developed countries, it results mainly in adverse economic effects on producers; in the USA alone, A. flavus contamination of crops results in more than a billion dollars in losses per year [41]. Current methods are insufficient to control the severe detrimental effects caused by AF contamination. This has led to efforts to identify novel genes that regulate AF biosynthesis in A. flavus that can in turn serve as targets in strategies designed to control AF contamination by this mycotoxigenic fungus. Our current study revealed that $\mathrm{AFB}_{1}$ production is regulated by $m t f A$ in $A$. flavus. The $m t f A$ gene encodes a putative $\mathrm{C}_{2} \mathrm{H}_{2}$ zinc finger domain-type transcription factor first described in the model fungus A. nidulans [20]. Interestingly, as in the case of ST in A. nidulans, wild-type levels of $m t f A$ expression are required to maintain normal AF biosynthesis. Both deletion and forced overexpression of $m t f A$ led to a decrease in AF levels compared to the controls in A. flavus-infected seeds. The decrease in AF accumulation was more dramatic when this gene was overexpressed; in this case, AF was only present in trace amounts or absent. The negative effect of $m t f A$ overexpression on AF production was also observed when the fungus was growing in a laboratory medium, where this decrease coincided with a reduction in the expression of aflR, encoding a transcription factor that acts as a specific AF cluster activator [14-19]. Reduction of aflR expression led to a reduction in the expression of the AF gene cluster, as indicated by the decrease in the expression of the structural gene ver1, used as a marker of cluster activation. The effect of the $m t f A$ deletion on AF appears to be substrate-dependent, since $\triangle m t f A$ showed similar levels of AF as the wild-type when growing on YGT medium, while a decrease was observed when $A$. flavus was growing on seed. This decrease in toxin production caused by the absence of $m t f A$ was also observed when ST was analyzed in the $A$. nidulans $m t f A$ deletion mutant on glucose minimum medium [20]. Furthermore, the remarkably strong repressing effect of $m t f A$ overexpression on toxin production was common in both fungi in all culture conditions assayed. Although alterations 
in $m t f A$ expression did not prevent fungal colonization of the seeds, the resulting dramatic decrease or blockage of $\mathrm{AFB}_{1}$ production could be relevant for the development of new control methodologies to prevent AF contamination of crops.

Interestingly, our study also indicated that in addition to $\mathrm{AF}$, the synthesis of many other compounds may also be affected by $m t f A$, indicating a broader $m t f A$ regulatory impact on $A$. flavus secondary metabolism. This broader regulatory scope is conserved in other Aspergillus species. In A. nidulans, besides ST, $m t f A$ regulates the synthesis of Penicillin and Terriquinone A [20]. Furthermore, recent transcriptome analyses of $m t f A$ in $A$. nidulans and A. fumigatus revealed hundreds of genes under $m t f A$ regulation, including secondary metabolite gene clusters [21]. It is likely that $m t f A$ might have a similar effect on the A. flavus genome, governing the activation of other gene clusters, besides the AF cluster, directing the synthesis of multiple compounds, as indicated by our chemical analyses of both laboratory medium and infected seeds.

Fungal secondary metabolism has been shown to be genetically linked to morphological development [42]. As in the case of $A$. nidulans and A. fumigatus, the absence of $m t f A$ in $A$. flavus affected conidiation; however, the effects observed on asexual development vary in different Aspergillus species. In the model fungus $A$. nidulans, deletion of $m t f A$ resulted in a strain with reduced conidial production [20]. This was also observed in $A$. flavus $\Delta m t f A$ when growing on live seeds; however, when growing on synthetic culture medium colonies, hyper-conidiation, accompanied by an increase in the expression of $b r l A$, a gene essential for conidiophore formation, was observed [39,40]. Overexpression of $m t f A$ led to a significant decrease in $b r l A$ transcription and conidial production on laboratory medium, as was also observed in A. fumigatus [22], whereas this effect was not detected when A. flavus was growing on seeds. This suggests that $m t f A$ regulation of conidiation is responsive to environmental conditions in A. flavus, and it varies in different fungal species. This species-dependent variation of the $m t f A$ regulatory output in developmental events is in agreement with our previous $m t f A$ comparative transcriptome analysis of $A$. nidulans and A. fumigatus [21], in which this divergent regulation of $m t f A$ in development was noted, suggesting that certain regulatory circuit rewiring has occurred through evolutionary processes.

Previously, $m t f A$ was shown to be necessary for cleistothecial production in A. nidulans [20]. Sclerotia have been shown to be vestigial structures of cleistothecia [43-45], and in some cases, ascospore-bearing ascocarps embedded within sclerotia (termed stromata) of A. flavus and A. parasiticus have been found [46,47]. This common origin between cleistothecia and sclerotia suggests that conserved genetic regulatory pathways controlling cleistothecial formation could also affect sclerotial production. Our study showed that sclerotial formation was also influenced by $m t f A$. Although sclerotia were produced in the absence of $m t f A$, their size was reduced in comparison to the wild-type. It is possible that this size reduction could affect the survival of these resistance structures under adverse conditions in the field.

During crop colonization, A. flavus produces a variety of extracellular hydrolytic enzymes to obtain nutrients from the host $[17,35,36,38,48]$. For this reason, we also examined whether $m t f A$ has a role in regulating lipase, amylase and protease activity in the secretome. Our results indicated that $m t f A$ has a minor effect on these activities; only a small reduction in lipase and protease activity was observed in the absence of this transcription factor. This coincided with similar colonization levels by all of the strains tested, as indicated by ergosterol levels measured in infected seeds. A slight increase in lipase activity was detected over time in the OEmtfA strain with respect to the wild-type. It is possible that this could be associated with the fact that seeds colonized by the A. flavus strain overexpressing $m t f A$ also showed a slightly faster pace in seed colonization and maceration compared to those infected with the wild-type.

In conclusion, our studies indicated that $m t f A$ affects several cellular processes in the agriculturally-important fungus $A$. flavus, influencing development in response to the environment, and in this way, affecting dissemination and survival of this fungal species. However, the most relevant finding in this study is the drastic decrease or suppression of AF production on infected live seeds 
achieved by altering the expression pattern of the $m t f A$ gene, making $m t f A$ a desirable candidate to target in control strategies against $\mathrm{AF}$ contamination of crops by A. flavus. In addition, we showed that $m t f A$ not only affects AF production in this fungus, but it also appears to govern the synthesis of other secondary metabolites. This is the first study of $m t f A$ homologs in an opportunistic plant pathogen. However, the fact the $m t f A$ is conserved in numerous Ascomycetes, together with its broad effect on secondary metabolism observed in Aspergillus species, suggests that an $m t f A$-based control approach could also be used to reduce the detrimental effects of other mycotoxigenic fungi, including other plant pathogens that endanger our food commodities.

Acknowledgments: We thank Baozhu Guo for providing us with the NC94022 Virginia peanut line used in this study. We would also like to thank Sourabh Dhingra for his technical support. This work was supported by USDA Grant 58-6435-4-015, the Department of Biological Sciences at NIU and the Program for Cultivation of Outstanding Youth Science and Technology Talents at Fujian Agriculture and Forestry University (xjq201410).

Author Contributions: Z.Z. and A.M.C. conceived and designed the experiments; Z.Z., J.M.L. and T.S. performed the experiments; Z.Z., J.M.L., T.S. and J.W.C. analyzed the data; J.W.C. and A.M.C. contributed reagents/materials/analysis tools; Z.Z. and AMC wrote the paper.

Conflicts of Interest: The authors declare no conflict of interest.

\section{References}

1. Bennett, J.W.; Klich, M. Mycotoxins. Clin. Microbiol. Rev. 2003, 16, 497-516. [CrossRef] [PubMed]

2. Perrone, G.; Susca, A.; Cozzi, G.; Ehrlich, K.; Varga, J.; Frisvad, J.C.; Meijer, M.; Noonim, P.; Mahakarnchanakul, W.; Samson, R.A. Biodiversity of Aspergillus species in some important agricultural products. Stud. Mycol. 2007, 59, 53-66. [CrossRef] [PubMed]

3. Dvorackova, I.; Kusak, V. Hepatocellular Carcinoma (a 28-year necropsy review). J. Pathol. Environ. Toxicol. Oncol. 1990, 10, 220-224.

4. Payne, G.A.; Brown, M.P. Genetics and Physiology of Aflatoxin Biosynthesis. Annu. Rev. Phytopathol. 1998, 36, 329-362. [CrossRef] [PubMed]

5. Probust, C.; Schulthess, F.; Cotty, P.J. Impact of Aspergillus section Flavi community structure on the development of lethal levels of aflatoxins in Kenyan maize (Zea mays). J. Appl. Microbiol. 2010, 108, 600-610. [CrossRef] [PubMed]

6. Sweeny, M.J.; Dobson, A.D. Molecular Biology of Mycotoxin Biosynthesis. FEMS Microbiol. Lett. 1999, 175, 149-163. [CrossRef]

7. Trail, F.; Mahanti, N.; Linz, J.E. Molecular Biology of Aflatoxin Biosynthesis. Microbiology 1995, 141(Pt 4), 755-765. [CrossRef] [PubMed]

8. Amaike, S.; Keller, N.P. Distinct Roles for VeA and LaeA in Development and Pathogenesis in A. flavus. Eukaryot Cell 2009, 8, 1051-1060. [CrossRef] [PubMed]

9. Cary, J.W.; Ehrlich, K.C.; Kale, S.P.; Calvo, A.M.; Bhatanagar, D.; Clevelend, T.E. Regulatory elements in aflatoxin biosynthesis. Mycotoxin Res. 2006, 22, 105-109. [CrossRef] [PubMed]

10. Cary, J.W.; Harris-Coward, P.Y.; Ehrlich, K.C.; Mack, B.M.; Kale, S.P.; Larey, C.; Calvo, A.M. NsdC and NsdD Affect Aspergillus flavus Morphogenesis and Aflatoxin Production. Eukaryot Cell 2012, 11, 1104-1111. [CrossRef] [PubMed]

11. Chang, P.K.; Scharfenstein, L.L.; Ping, L.; Ehrlich, K. Aspergillus flavus VelB acts distinctly from VeA in conidiation and may coordinate with FluG to modulate sclerotial production. Fungal Genet Biol 2013, 58, 71-79. [CrossRef] [PubMed]

12. Duran, R.M.; Cary, J.W.; Calvo, A.M. Production of cyclopiazonic acid, aflatrem, and aflatoxinby Aspergillus flavus is regulated by veA, a gene necessary for sclerotial formation. Appl. Microbiol. Biotechnol. 2007, 73, 1158-1168. [CrossRef] [PubMed]

13. Duran, R.M.; Cary, J.W.; Calvo, A.M. The role of veA on Aspergillus flavus infection of peanuts, corn and cotton. Open Mycol. J. 2009, 3, 27-36. [CrossRef]

14. Brown, D.W.; Yu, J.H.; Kelkar, H.S.; Fernandes, M.; Nesbitt, T.C.; Keller, N.P.; Adams, T.H.; Leonard, T.L. Twenty-five coregulated transcripts define a sterigmatocystin gene cluster in Aspergillus nidulans. Proc. Natl. Acad. Sci. USA 1996, 93, 1418-1422. [CrossRef] [PubMed] 
15. Fernandes, M.; Keller, N.P.; Adams, T.H. Sequence-specific binding by Aspergillus nidulans AflR, a C-6 zinc cluster protein regulating mycotoxin biosynthesis. Mol. Microbiol. 1998, 28, 1355-1365. [CrossRef] [PubMed]

16. Payne, G.A.; Nystrom, G.J.; Bhatnagar, D.; Cleveland, T.E.; Woloshuk, C.P. Cloning of the afl-2 gene involved in aflatoxin biosynthesis from Aspergillus flavus. Appl. Environ. Microbiol. 1993, 59, 156-162. [PubMed]

17. Woloshuk, C.P.; Yousibova, G.L.; Rollins, J.A.; Bhatnagar, D.; Payne, G.A. Molecular Characterization of the afl-1 Locus in Aspergillus flavus. Appl. Enviorn. Microbiol. 1995, 61, 3019-3023.

18. Yabe, K.; Nakajima, H. Enzyme Reactions and Genes in Aflatoxin Biosynthesis. Appl. Microbiol. Biotechnol. 2004, 64, 745-755. [CrossRef] [PubMed]

19. Yu, J.H.; Butchko, R.A.; Fernandes, M.; Keller, N.P.; Leonard, T.J.; Adams, T.H. Conservation of structure and function of the aflatoxin regulatory gene aflR from Aspergillus nidulans and A. flavus. Curr. Genet. 1996, 29, 549-555. [CrossRef] [PubMed]

20. Ramamoorthy, V.; Dhingra, S.; Kincaid, A.; Shantappa, S.; Feng, X.; Calvo, A.M. The putative C2H2 transcription factor $M t f A$ is a novel regulator of secondary metabolism and morphogenesis in Aspergillus nidulans. PLoS ONE 2013, 8, e74122.

21. Lind, A.; Wisecaver, J.H.; Smith, T.D.; Feng, X.; Calvo, A.M.; Rokas, A. Examining the evolution of the regulatory circuit controlling secondary metabolism and development in the fungal genus Aspergillus. PLoS Genet. 2015, 11, e1005096. [CrossRef] [PubMed]

22. Smith, T.D.; Calvo, A.M. The $m t f A$ transcription factor gene controls morphogenesis, gliotoxin production, and virulence in the opportunistic human pathogen Aspergillus fumigatus. Eukaryot Cell 2014, 13, 766-775. [CrossRef] [PubMed]

23. Comera, C.; André, K.; Laffitte, J.; Collet, X.; Galtier, P.; Maridonneau-Parini, I. Gliotoxin from Aspergillus fumigatus affects phagocytosis and the organization of the actin cytoskeleton by distinct signaling pathways in human neutrophils. Microbes Infect. 2007, 9, 47-54. [CrossRef] [PubMed]

24. Kwon-Chung, K.J.; Sugui, J.A. What do we know about the role of gliotoxin in the pathobiology of Aspergillus fumigatus? Med. Mycol. 2009, 47, S97-S103. [CrossRef] [PubMed]

25. Piva, T.J. Gliotoxin induces apoptosis in mouse 1929 fibroblast cells. Biochem. Mol Biol. Int. 1994, 33, 411-419. [PubMed]

26. Stanzani, M.; Orciuolo, E.; Lewis, R.; Kontoyiannis, D.P.; Martins, S.L.; St John, L.S.; Komanduri, K.V. Aspergillus fumigatus suppresses the human cellular immune response via gliotoxin-mediated apoptosis of monocytes. Blood 2005, 105, 2258-2265. [CrossRef] [PubMed]

27. Yoshida, L.S.; Abe, S.; Tsunawaki, S. Fungal gliotoxin targets the onset of superoxide-generating NADPH oxidase of human neutrophils. Biochem. Biophys. Res. Commun. 2000, 268, 716-723. [CrossRef] [PubMed]

28. Wu, T.S.; Linz, J.E. Recombinational inactivation of the gene encoding nitrate reductase in Aspergillus parasiticus. Appl. Environ. Microbiol. 1993, 59, 2998-3002. [PubMed]

29. Cary, J.W.; Han, Z.; Yin, Y.; Lohmar, J.M.; Shantappa, S.; Harris-Coward, P.Y.; Mack, B.; Ehrlich, K.C.; Wei, Q.; Arroyo-Manzanares, N.; et al. Transcriptome analysis of Aspergillus flavus reveals veA-dependent regulation of secondary metabolite gene clusters, including the novel aflavarin cluster. Eukaryot Cell 2015, 10, 983-997.

30. Szewczyk, E.; Nayak, T.; Oakley, E.C.; Edgerton, H.; Xiong, Y.; Naimeh, T.-T.; Osmani, S.A.; Oakley, B.R. Fusion PCR and gene targeting in Aspergillus nidulans. Nat. Protoc. 2006, 1, 3111-3121. [CrossRef] [PubMed]

31. Steinbach, W.J.; Cramer, R.A.; Perfect, B.Z.; Henn, C.; Nielsen, K.; Heitman, J.; Perfect, J.R. Calcineurin inhibition or mutation enhances cell wall inhibitors against Aspergillus fumigatus. Antimicrob. Agents Chemother. 2007, 51, 2979-2981. [CrossRef] [PubMed]

32. Cary, J.W.; Ehrlich, K.C.; Bland, J.M.; Montalbano, B.G. The aflatoxin biosynthesis cluster gene, aflX, encodes an oxidoreductase involved in conversion of vesicolorin A to demethylsterigmatocystin. Appl. Environ. Microbiol. 2005, 72, 1096-1101. [CrossRef] [PubMed]

33. Sambrook, J.; Russell, D.W. Molecular Cloning: A Laboratory Manual, 3rd ed.; Cold Spring Harbor Laboratory Press: Cold Spring Harbor, NY, USA, 2001.

34. Livak, K.J.; Schmittgen, T.D. Analysis of relative gene expression data using real-time quantitative PCR and the 2(-Delta Delta C(T)) method. Methods 2001, 24, 402-408. [CrossRef] [PubMed]

35. Dolezal, A.L.; Obrian, G.R.; Nielsen, D.M.; Woloshuk, C.P.; Boston, R.S.; Payne, G.A. Localization, morphology and transcriptional profile of Aspergillus flavus during seed colonization. Mol. Plant Pathol. 2013, 14, 898-909. [CrossRef] [PubMed] 
36. Mellon, J.E.; Cotty, P.J.; Dowd, M.K. Aspergillus flavus hydrolases: Their roles in pathogenesis and substrate utilization. Appl. Microbiol. Biotechnol. 2007, 77, 497-504. [CrossRef] [PubMed]

37. Amaike, S.; Affeldt, K.J.; Yin, W-B.; Franke, S.; Choithani, A.; Keller, N.P. The bZIP Protein MeaB Mediates Virulence Attributes in Aspergillus flavus. PLoS ONE 2013, 8, e74030. [CrossRef] [PubMed]

38. Duran, R.M.; Gregerson, S.; Smith, T.D.; Behtariya, P.J.; Cary, J.W.; Harris-Coward, P.Y.; Mattison, C.P.; Grimm, C.; Calvo, A.M. The Role of Aspergillus flavus veA in the production of extracellular proteins during the growth on starch substrates. Appl. Microbiol. Biotechnol. 2014, 98, 5081-5094. [CrossRef] [PubMed]

39. Adams, T.H.; Boylan, M.T.; Timberlake, W.E. brlA is necessary and sufficient to direct conidiophore development in Aspergillus nidulans. Cell 1988, 54, 353-362. [CrossRef]

40. Han, S.; Adams, T.H. Complex control of the developmental regulatory locus brlA in Aspergillus nidulans. Mol. Genet. Genom. 2001, 266, 260-270.

41. Vardon, P.; McLaughlin, C.; Nardinelli, C. Potential Economic Costs of Mycotoxins in the UnitedStates. In Mycotoxins: Risks in Plant, Animal, and Human Systems; Council for Agricultural Science and Technnology (CAST): Ames, IA, USA, 2003.

42. Calvo, A.M.; Wilson, R.A.; Bok, J.W.; Keller, N.P. Relationship between secondary metabolism and fungal development. Microbiol. Mol Biol. Rev. 2002, 66, 447-459. [CrossRef] [PubMed]

43. Geiser, D.M.; Timberlake, W.E.; Arnold, M.L. Loss of Meiosis in Aspergillus. Mol. Biol. Evol. 1996, 13, 809-817. [CrossRef] [PubMed]

44. Ramirez-Prado, J.H.; Moore, G.G.; Horn, B.W.; Carbone, I. Characterization and Population Analysis of the Mating-Type Genes in Aspergillus flavus and Aspergillus parasiticus. Fungal Genet. Biol. 2008, 45, 1292-1299. [CrossRef] [PubMed]

45. Wada, R.; Maruyama, J.; Yamaguchi, H.; Yamamoto, N.; Wagu, Y.; Paoletti, M.; Archer, D.B.; Dyer, P.S.; Kitamoto, K. Presence and functionality of mating type genes in the supposedly asexual filamentous fungus Aspergillus oryzae. Appl. Environ. Microbiol. 2012, 78, 2819-2829. [CrossRef] [PubMed]

46. Horn, B.W.; Moore, G.G.; Carbone, I. Sexual reproduction in Aspergillus flavus. Mycologia 2009, 101, $423-429$. [CrossRef] [PubMed]

47. Horn, B.W.; Sorensen, R.B.; Lamb, M.C.; Sobolev, V.S.; Olarte, R.A.; Worthington, C.J.; Carbone, I. Sexual reproduction in Aspergillus flavus sclerotia naturally produced in corn. Phytopathology 2014, 104, 75-85. [CrossRef] [PubMed]

48. Cotty, P.J.; Cleveland, T.E.; Brown, R.L.; Mellon, J.E. Variation in polygalacturonase production among Aspergillus flavus isolates. Appl. Environ. Microbiol. 1990, 56, 3885-3887. [PubMed]

(C) 2016 by the authors; licensee MDPI, Basel, Switzerland. This article is an open access article distributed under the terms and conditions of the Creative Commons by Attribution (CC-BY) license (http://creativecommons.org/licenses/by/4.0/). 